\title{
Postoperative Nausea and Vomiting: Risks, Prophylaxis, Non-Drug Alternative Methods
}

\author{
Ebru Çanakç1 ${ }^{1}$, Tuba Çatak ${ }^{1}$ \\ ${ }^{1}$ Ordu University, School of Medicine, Department of Anesthesiology and Reanimation, Ordu, Turkey
}

Received: 23 October 2019, Accepted: 25 November 2019, Published online: 31 December 2019 (C) Ordu University Institute of Health Sciences, Turkey, 2019

\begin{abstract}
Nausea and vomiting are among the most common complaints in the postoperative period. The type of surgical technique and the site of intervention, as well as the anesthetic drugs and methods directly contribute to the rates of postoperative nausea and vomiting (PONV). One of the most important factors affecting the frequency of postoperative nausea and vomiting is the anesthetic drugs and methods used. Many antiemetic drugs are used to prevent postoperative nausea and vomiting. With the use of these drugs, side effects especially such as delayed recovery from anesthesia, dry mouth, undesirable changes in blood pressure and the occurrence of extrapyramidal symptoms are seen. Several antiemetic drugs are commonly used for the treatment of postoperative nausea and vomiting; including scopolamine, chlorpromazine, diphenhydramine, metoclopramide, promethazine, and ondansetron. Low-dose haloperidol and metoclopramide are effective in opioid-induced nausea and vomiting. Dexamethasone reduces the incidence of postoperative nausea in the first 6 hours after surgery. Oral intake starts in patients with severe vomiting parenteral fluid and electrolyte treatment is required. Sometimes provide may need gastric decompression with nasogastric tube. Parenteral fluid and electrolyte treatment and in severe cases gastric decompression with a nasogastric tube may be necessary. Alternative medicine such as acupuncture and acupressure can help prevent or reduce postoperative nausea and vomiting. The aim of this review is to examine the medical therapies and drug-free methods used for the treatment of PONV in the light of the literatüre
\end{abstract}

Key words: postoperative nausea, vomiting, drug, acupuncture

Suggested Citation: Canakci E, Catak T. Postoperative Nausea and Vomiting: Risks, Prophylaxis, Non-Drug Alternative Methods. Middle Black Sea Journal of Health Science, 2019; 5(3):284-290

\section{Address for correspondence/reprints:}

Ebru Çanakçı

Telephone number: +90 (532) 2651687

E-mail: canakciebru@gmail.com

DOI: $10.19127 / \mathrm{mbsjohs.637394}$

\section{Introduction:}

Postoperative nausea and vomiting (PONV) are the most frequent early complications of general anesthesia, causing dissatisfaction in the anesthesiologist and the patient. A considerable number of patients describe PONV among the most discomforting postoperative complaints, even more unpleasant than pain (Macario et al.,1999; Macario, Weinger, Truong et al.1999; Gan et al.2003). In the literature, the incidence of PONV is reported to be $25-30 \%$ in the absence of pharmacological treatments and $70 \%$ in high-risk patients in the general population. Despite these high rates, completely effective prevention and treatment strategies have not been established for PONV, yet 
(Huang et al.,2001; Gan et al.,2003). However, several risk factors and preoperative prophylactic antiemetic treatment strategies have been defined in recent studies and published guidelines (Apfel et al.,1999; Gan et al.,2003; McCracken et al.,2008). The reported risk factors of PONV include the type of surgery, female gender, being a non-smoker, previous experiences of PONV or motion sickness, and postoperative opioid use (Apfel et al.,1997; Myklejord et al.,2012). In the guidelines published in 2003 and 2008; symptomatic treatment with antiemetics is recommended for low-risk patients, prophylactic antiemetic use is recommended only for the treatment of moderate-to-high-risk patients, and drug combinations are recommended only for the high-risk patients (Gan et al.,2003; McCracken et al.,2008). For preventing the development of PONV, different classes of antiemetics are used; including dexamethasone, 5-hydroxytryptamine, serotonin (5-HT3) receptor antagonists, and dopamine receptor antagonists. These pharmacological agents have their mechanisms of action independent from each other. It is reported that the efficacy of antiemetics is limited in monotherapy but enhanced in combined use (Henzi et al., 2000; Apfel et al., 2004). Particularly, the combined use of dexamethasone with 5-HT3 receptor antagonists is known to be associated with an enhanced antiemetic activity (Eberhart et al., 2000; Henzi I, Walder B et al., 2000).

The aim of this article is to review the pharmacological and drug-free methods used for the treatment of PONV in the light of the literature.

\section{Definition of Postoperative Nausea and Vomiting and General Information:}

Complaints of nausea and vomiting developing in the first 24 hours after surgical intervention are defined as postoperative nausea and vomiting (PONV). PONV is a common condition associated with the administration of anesthesia, leading to patient dissatisfaction. It may sometimes become severe enough to contribute to intolerably high heart rates or blood pressure, increased intrathoracic pressure, and central venous pressure. PONV is reported at rates as high as $80 \%$ in high-risk patients and at a $30 \%$ rate in the general population (Grabowska-Gaweł et al.,2006). The most common patient-related risk factors include being a middleaged woman, a non-smoking woman, and having a history of PONV or motion sickness. The treatmentassociated factors to develop PONV include the use of volatile anesthetics (inhalation anesthetic agents), prolonged surgery, $\mathrm{N}_{2} \mathrm{O}$ use, and administration of postoperative opioids. The type and the site of surgery are among the major players, too; with ocular, tympanic, intracranial, abdominal, and gynecologic operations posing a high risk to develop PONV (Grabowska-Gaweł et al.,2006). Grabowska-Gaweł et al. study on patients receiving general anesthesia reported that nausea and vomiting were observed in $22.7 \%$ and $13.2 \%$ of the patients respectively and that $14.2 \%$ of the patients had developed both symptoms (Grabowska-Gawel et al.,2006).

\subsection{Pathophysiology}

The pathophysiology of nausea and vomiting involves complex mechanisms and has not been completely understood, yet. Nausea is a conscious sensation and vomiting is a complex reflex. The former requires the involvement of cortical structures and the latter is controlled by the medulla oblongata. The act of vomiting requires coordination of systems including the involvement of emesis-related afferents combined with the actions of the respiratory, gastrointestinal, and abdominal muscles. This whole process is mediated by a specific area in the central nervous system, namely the vomiting center. The current hypothesis suggests that there are neurons in the medulla oblongata, specifically organized for being activated by the nausea center; which coordinates nausea and emesis to occur sequentially. This hypothesis favors the concept of a "central stimulus pattern", suggesting that it is more acceptable than the "vomiting center" concept (Aygin et al.,2004). Even though the nucleus tractus solitarius (NTS) and other specific nuclei of the reticular formation (including the respiratory nuclei) are considered to be the important sites for generating emesis, the central stimulus pattern is not well defined (Aygin et al.,2004). It is reported that NTS activates the central stimulus pattern and surrounding neuron groups to generate the autonomic and motor responses leading to vomiting (Çekmen et al.,2003).

The following anatomical structures are mainly involved in projectile vomiting;

1. The vagal afferent fibers of the gastrointestinal tract

2. The vestibular system

3. The cerebral cortex, thalamus, and hypothalamus

4. The area postrema (AP) 
Enteroendocrine cells induce serotonin synthesis when circulating drugs or toxins are present in the system. The synthesis of serotonin stimulates the vagal afferent fibers of the gastrointestinal tract. Other types of emesis, psychogenic vomiting, and vomiting induced by smelling or visual stimuli are generated by the stimulation of thalamus, hypothalamus, the respective areas in the cortex, and the vestibular nuclei; which receive motiondependent sensory data (Aygin et al.,2004).

Recent research has focused on AP and the chemoreceptor trigger zone (CTZ). AP is a specific area located at the base of the fourth ventricle in the medulla oblongata. AP is rich in blood vessels, but it does not have a specific blood-brain barrier. These properties render the chemoreceptors in the AP highly sensitive to the effects of circulating emetogenic agents in the blood and cerebrospinal fluid. Therefore, AP plays an important role in druginduced vomiting. AP receives stimuli from the emetic receptors in a variety of ways; including the transmission of stimuli via the direct afferents, and the visceral vagus nerve. Molecules in the blood circulation and autonomic stimuli transmitted by the descending pathways from the hypothalamus stimulate AP, too (Aygin et al.,2004). Consequently, the vagus nerve and the neuron groups associated with nausea and vomiting are activated by the afferent fibers and interaction of various neurotransmitters; which are serotonin (5HT), dopamine (D), histamine (H), Substance P, acetylcholine, and opioids. The receptors of these neurotransmitters are available in the vagal afferents (5-HT3 receptors), vestibular nucleus (M3/M5 muscarinic acetylcholine receptors and H1 receptors), AP (M, 5-HT3-, and D2-opioid receptors), and NTS (M receptors, 5-HT3, neurokinin-1, and Substance P receptors) (Aygin et al.,2004).

Disadvantages of PONV include the discomfort in the patient, prolonged length of hospital stay, and delays in recovery (Çekmen et al.,2003). Besides these untoward consequences, severe PONV is associated with consequent fluid and electrolyte loss, aspiration into airways, contamination of the surgical site, increased strain in the sutured incision site, and increased intraocular and intraabdominal pressure (Kayhan Z ,2004; Wesmiller et al.,2017).

The antagonization of neuromuscular blockade, which was previously thought to be a risk factor for PONV, showed that obesity and anxiety were not associated with PONV (Gan et al.,2014). Postoperative pain, hypotension, capacity for movement and fluid intake may also predispose to
PONV (Ozatamer et al.,2010). Simplified risk models have been developed in order to calculate the PONV risks of adult patients (The Apfel score, Table 1). In the absence of any risk factors, the PONV risk is assumed to be $10 \%$. While the female gender, non-smoking, history of PONV/motion sickness, and postoperative opioid use is associated with a $20 \%$ risk for PONV, it is accepted that the risk increases by $20 \%$ with the addition of each risk factor and reaches $80 \%$ in the presence of the four factors (Gan et al.,2014) (Table 1).

Table 1. Scoring system to predict postoperative nausea and vomiting in adults (The Apfel Scoring System)

\begin{tabular}{lc}
\hline Risk factor & Scoring \\
\hline Female gender & 1 \\
Non-smoking & 1 \\
History of PONV/Motion sickness & 1 \\
Postoperative opioid use & 1
\end{tabular}

The total Apfel scores may range from 0 to 4 (Gan et al.,2014).

Risk factors for PONV can be categorized under three main titles as follows:

1. Patient-dependent risk factors: Female gender, history of previous PONV, history of smoking, history of motion sickness, and age.

2. Anesthesia-dependent risk factors: Receiving inhalation anesthetics, duration of anesthesia, nitric oxide use, and postoperative opioid use

3. Surgery-dependent factors: Cholecystectomy, laparoscopic surgical procedures, and gynecological surgery (Diemunsch et al.,2007).

\subsection{Evaluation of Postoperative Nausea and Vomiting}

It is extremely difficult to evaluate PONV objectively since these complaints are subjective experiences of the patients. However, the Abramowitz Emesis Scoring system may help grade the severity of vomiting (Abramowitz et al.,1983) (Table 2). 
Table 2. Abramowitz Emesis Scoring System

\begin{tabular}{lc}
\hline Evaluation & Score \\
\hline No vomiting & 0 \\
Slight vomiting (1 times) & 1 \\
Moderate vomiting (2 times) & 2 \\
Severe vomiting (4 times) & 3 \\
Persistent vomiting & 4 \\
\hline
\end{tabular}

\subsection{Treatment}

The treatment and prevention of PONV are the major components of the management of these complaints. Even though some anesthetists prefer to administer routine prophylaxis to the surgical patients to prevent PONV; this strategy brings along additional costs as well as the risks of administrating extra drugs. A prophylactic strategy can be the administration of a balanced crystalloid solution as dehydration in the perioperative period is an important risk factor to develop PONV. However, it is more critical to perform an individual risk evaluation in each patient instead of administering routine prophylactic management strategies (Table 3) (Apfel et al.,2012).

Table 3. Risk evaluation with the Apfel scoring in terms of PONV incidence

\begin{tabular}{|cc|}
\hline Score & PONV Incidence \\
\hline $\mathbf{0}$ & 10 \\
$\mathbf{1}$ & 21 \\
$\mathbf{2}$ & 39 \\
$\mathbf{3}$ & 61 \\
$\mathbf{4}$ & 79 \\
\hline
\end{tabular}

Most patients receiving prophylactic drug treatment to prevent PONV do not benefit and 1$5 \%$ of these patients may experience mild side effects such as headache, sedation or dry mouth (Carlisle et al.,2006). If vomiting occurs despite the prophylactic management strategies, it will mean that the pharmacological agents used for prophylaxis will not be effective for the treatment (Kovac et al.,1999).

Kappen et al. conducted a single center randomized study on 12,032 patients and demonstrated the efficacy of the risk scoring system in identifying the patients to administer prophylactic treatment for PONV (Kappen et al.,2014). Antiemetic prophylaxis is recommended for moderate and severe risk patients to develop PONV. If two drugs are to be used in combination for the prophylaxis, they should be selected from different pharmacological classes. Combination treatments are usually reported to be more effective than monotherapy (De Oliveira et al.,2011).

Although the type of surgery is an important risk factor in the development of postoperative nausea and vomiting, it has not yet been proven to be an independent risk factor. The incidence is higher in some types of surgery (e.g. abdominal surgeries) due to prolonged exposure to general anesthesia and high-dose opioid use. Recent studies have shown that laparoscopic, gynecologic and cholecystectomy operations in terms of PONV are high-risk surgeries In summary, surgical-related factors and anesthesiarelated factors can create postoperative nausea and vomiting with a combined effect (Apfel et al.,2012) The drugs used to treat PONV are briefly mentioned in the following section.

\subsection{Drugs Used in PONV Treatment}

\subsubsection{5-Hydroxytryptamine (5-HT3) antagonists}

Ondansetron, granisetron, dolasetron are the 5HT3 agonists and they are used in daily doses of 4$8 \mathrm{mg}$. These pharmacological agents act completely in CTZ, inhibiting the 5-HT3 receptors. Common side effects of these medications include headaches, flushing, constipation, elevations in the levels of liver enzymes, and bradycardia. These medications are recommended to be given to patients at the end of surgery (De Oliveira et al.,2012). Ondansetron is reported as the gold standard treatment option in many studies in the literature (Jokela et al.,2009).

\subsubsection{Dexamethasone}

Dexamethasone is an effective antiemetic agent at daily doses of 4-8 $\mathrm{mg}$. It is recommended to administer dexamethasone immediately after the induction of anesthesia to obtain the maximum effect. The administration of rapid bolus doses of dexamethasone to awakened patients may cause irritability, perineal pain, and burning sensation. Other common postoperative side effects of dexamethasone include euphoria and insomnia (De Oliveira et al.,2012).

\subsubsection{Cyclizine}

Cyclizine is the only pharmacological agent with H1 antagonist activity displaying antiemetic properties. Cyclizine is usually recommended to be administered at a daily dose of $50 \mathrm{mg}$. Cyclizine has 
been in use for a long time with a well-established safety and efficacy profile; however, it is not devoid of some serious adverse effects limiting its use. These adverse events include flushing and tachycardia, injection pain, and phlebitis (Habib et al.,2004).

\subsubsection{Phenothiazines}

Prochlorperazine and promethazine are the pharmacological agents in this class. Their use for the treatment of PONV is limited due to their side effect profile due to the antidopaminergic and anticholinergic side effects. Short-term administration of these medications is most commonly associated with extrapyramidal symptoms (EPS), acute dystonia, and sedation (antidopaminergic effects). The use of these medications should be avoided in patients with Parkinson's disease. Anticholinergic effects manifest with dry mouth and tachycardia. The effective dose of prochlorperazine is reported to be $12.5 \mathrm{mg}$ administered by an intramuscular injection for salvage treatment or prophylactic treatment of PONV (Schaub et al.,2012).

\subsubsection{Droperidol}

Droperidol antagonizes the dopamine D2 receptors in CTZ. Droperidol is available as a 2.5 $\mathrm{mg} / \mathrm{ml}$ solution. The recommended dose for the antiemetic effect is $0.625-1.25 \mathrm{mg}$ to be administered at the end of the surgical intervention. However, cardiac complications are reported even at low doses. Other adverse events include anxiety and EPS, which are dose-dependent, and which may occur 12 hours after the administration (Lamond et al.,1998).

\subsubsection{Metoclopramide}

Metoclopramide is a weak D2 receptor antagonist in CTZ. Although the efficacy of metoclopramide has been reported at doses as high as $20-30 \mathrm{mg}$, a single $10 \mathrm{mg}$ dose has been shown to be effective after intravenous administration for the prophylactic treatment of PONV in the early period (Murphy et al.,2006). EPS may develop 72 hours after a single dose. Due to the risk of developing EPS, metoclopramide use needs to be avoided in young female patients (Murphy et al.,2006).

\subsubsection{Modes of administration of anesthetic agents}

Anesthetic agents can play a major role in the development of PONV, demonstrating different effects at different doses and modes of administration. For instance, a bolus administration of propofol for induction has not been associated with the development of PONV; however, total intravenous anesthesia has been shown to reduce the risk of PONV. Therefore, administration of total intravenous anesthesia is recommended in high-risk patients in combination with the use of appropriate antiemetics agents when indicated (Habib et al.,2006).

\subsubsection{Miscellaneous}

Opioid receptor antagonists, such as naloxone at very low doses $(0.25 \mathrm{mg} / \mathrm{kg} / \mathrm{h})$ have been demonstrated to reduce the risk of developing opioid-associated PONV after the use of opioids to obtain analgesia (Melzack et al.,1977). There is an increasing interest in neurokinin-1 (NK-1/substance $\mathrm{P})$ antagonists. The representing drug in this class is aprepitant, characterized by a low risk profile, favorable oral availability, and significant efficacy. These properties suggest the use of molecules in this pharmacological class as attractive options when appropriate. Combination of 5-HT3 and NK-1 receptor antagonists in the treatment for up to 48 hours are reported to reduce the PONV rates significantly (Habib et al.,2006).

\section{Acupuncture in PONV Treatment}

Acupuncture is applied to patients by stimulating the skin and subcutaneous muscle tissue. Acupuncture points are characterized by lower electrical resistance and higher electrical potential compared to the surrounding skin. This principle is used for locating the acupuncture points with the use of detectors. Studies on the bioelectrical principles of acupuncture have demonstrated that it is possible to accurately measure the differences in the levels of resistance and electrical potentials. It has been reported that these findings can be used for diagnostic purposes, too. Furthermore, almost 70$80 \%$ of the acupuncture points are associated with the trigger points and most of these points were found out to be identical with the motor points of the muscles (Liao et al.,1975; Bokhari et al.,1977). The P6 (Nei Guan) point is the most commonly used acupuncture point for the PONV treatment (Chernyak et al.,2005), located between the flexor carpi radialis and palmaris longus tendons on the middle finger line at an approximately $6 \mathrm{~mm}$ depth and 2 thumb breadths $(2 \mathrm{~cm})$ away from the inner curve of the wrist (Chernyak et al.,2005). The P-6 point shown in Figure 1 (Figure 1 taken from 
Guyton Physiology major text book;Guyton et al.,2001).

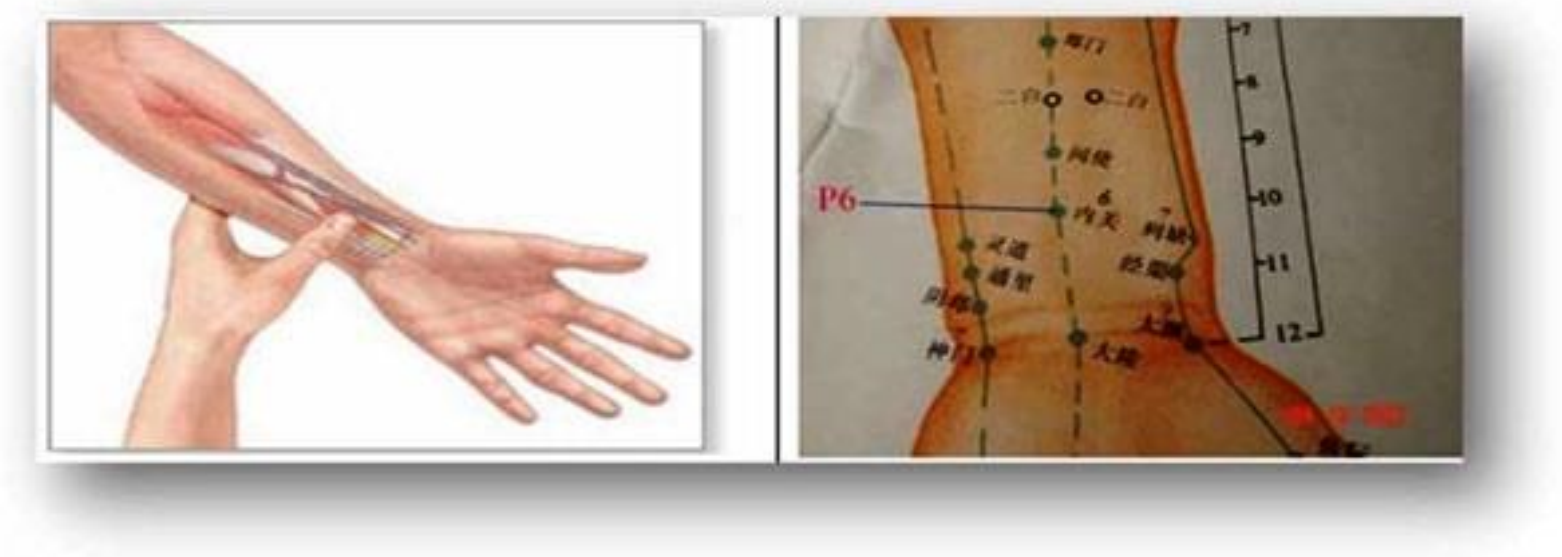

Figure 1. Anatomical Localization of P-6 Point

The efficacy of antiemetics is currently limited for the treatment of PONV. The combination of rare complications along with the relatively low efficacy of the pharmacological agents has increased interest in alternative medicine methods for PONV treatment (Dundee et al.,1986). Acupuncture is among the most commonly used and studied alternative medicine method in anesthesia for the treatment of PONV (Al-Sadi et al.,1997; Kotani et al.,2001; Hickman et al.,2005). Stimulation of the Nei Guan (Pericardium-6) point is effective in alleviating the severity of PONV (Al-Sadi et al.,1997; Wang et al.,2002; Hickman et al.,2005). It is more effective in preventing the development of early postoperative PONV compared to late postoperative PONV (Fassoulaki et al.,1993; Tavlan et al.,1996; Wang et al.,2002). Stimulation of this acupuncture point with electric current by using appropriate acupuncture techniques is reported to reduce the severity of postoperative nausea (Zarate et al;2001). The efficacy of stimulating the Nei Guan point in adults and children has been shown to be same as the ondansetron therapy in the treatment of PONV (Fassoulaki et al;1993; Rusy et al., 2002; White et al., 2002).

A variety of acupuncture stimulation methods have been tried for the treatment of postoperative nausea and vomiting. Some of these include the combination of acupuncture and acupressure, acupressure treatment alone, acupressure with electric stimulation, laser stimulation, and capsicum (Capsaicin) therapy (Tavlan et al.,1996; Shenkman et al.,1999). However, the best stimulation method has not been established, yet. Administration of non-invasive treatment methods like the aforementioned acupuncture stimulation techniques

is easier, painless, and better tolerated by the patient; however, the number of studies reporting inefficacy of these methods is considerable and studies in the literature report partial efficacy only on nausea but no efficacy on vomiting. Therefore, non-invasive methods have been suggested to be less effective and inadequate in the literature (Dundee et al., 1986; Tavlan et al., 1996).

\section{Conclusion}

PONV remains to be a factor contributing to morbidity and significantly deteriorating patient comfort. To prevent the morbidity, a risk classification should be performed to estimate the development and severity of PONV by evaluating the patient with the use of the reliable scoring systems in the preoperative period. A variety of perioperative and postoperative management strategies (such as the use of prophylactic antiemetic drugs, avoiding the use of opioids for analgesia, etc.) can be employed based on the results obtained by using the risk classification systems. Alternative methods such as acupuncture and acupressure can be administered for the treatment of PONV, too, promising efficacy with no side effects. We believe that it will very beneficial for anesthesiologists to be informed of the acupressure point P-6 (Nei Guan) to offer this highly simple, inexpensive, and side-effect-free alternative management strategy for the treatment and prophylaxis of PONV. 
Peer-review: Externally peer-reviewed.

Author Contributions: Concept- EC., DesignEC, TC., Supervision- EC, TC, Literature ReviewEC, TC, Writing- EC.., Critical Review- TC

Conflict of Interest: No conflict of interest was declared by the author.

Financial Disclosure: The author declared that this study hasn't received no financial support.

\section{References}

Abramowitz MD, Oh TH, Epstein BS, Ruttimann UE, Friendly DS.The antiemetic effect of droperidol following outpatient strabismus surgery in children. Anesthesiology. 1983;59(6):579-83.

Al-Sadi M, Newman B, Julious SA. Acupuncture in the prevention of postoperative nausea and vomiting. Anaesthesia 1997;52(7):658-61.

Apfel CC, Heidrich FM, Jukar-Rao S, Jalota L, Hornuss C, Whelan RP et al., Evidence-based analysis of risk factors for postoperative nausea and vomiting. Br J Anaesth 2012;109(5):742-53.

Apfel CC, Korttila K, Abdalla M, Kerger H, Turan A, Vedder I, et al. A factorial trial of sixinterventions for the prevention of postoperative nausea and vomiting. $\mathrm{N}$ Engl $\mathrm{J}$ Med. 2004; 350(24):2441-51.

Apfel CC, Laara E, Koivuranta M, Greim CA, Roewer N. A simplified risk score for predicting postoperative nausea and vomiting: conclusions from cross-validations between two centers. Anesthesiology 1999;91(3):693-700.

Aygin D, Nausea and Vomiting. Journal of Intensive Care Nursing 2004;20(1):44-56.

Bokhari F, Sawatzky JA. Chronic neuropathic pain in women after breast cancer treatment. Pain Manag Nurs 2009;10(4): 197-205.

Carlisle JB, Stevenson CA. Drugs for preventing postoperative nausea and vomiting. Cochrane Database Syst Rev 2006;19(3): CD004125

Chernyak GV, Sessler DI. Perioperative Acupuncture and Related Techniques. Anesthesiology 2005;102(5):1031-49; quiz 1077-8.

Çekmen N, Akçabay M, Mahlı A, Arslan M. Comparison of the Effects of Dexamethasone and Metoclopramide in Postoperative Nausea and Vomiting. Erciyes Medical Journal 2003;25(3):137-43.
De Oliveira GS, Almeida MD, Benzon HT, McCarthy RJ. Perioperative single dose systemic dexamethasone for postoperative pain: a meta-analysis of randomized controlled trials. Anesthesiology 2011;115(3):575-88.

De Oliveira GS, Castro-Alves LJ, Chang R, Yaghmour E, McCarthy RJ. Systemic metoclopramide to prevent postoperative nausea and vomiting: a meta-analysis without Fujii's studies. Br J Anaesth 2012;109(5):688-97.

Diemunsch P, Apfel C, Gan T J, Candiotti K, Philip B K, Chelly J et al., Preventing postoperative nausea and vomiting: post hoc analysis of pooled data from two randomized active-controlled trials of aprepitant. Curr Med Res Opin 2007;23(10): 2559-65.

Dundee JW, Chestnutt WN, Ghaly RG, Lynas AG. Traditional Chinese acupuncture: a potentially useful antiemetic? $\mathrm{Br}$ Med J (Clin Res Ed). 1986;293(6547):583-4.

Eberhart LH, Morin AM, Georgieff M. Dexamethasone for prophylaxis of postoperative nausea and vomiting. A metaanalysis of randomized controlled studies. Der Anaesthesist 2000;49(8):713-20.

Fassoulaki A, Papilas K, Sarantopoulos C, Zotou M. Transcutaneous electrical nevre stimulation reduces the incidence of vomiting after hysterectomy. Anesth Analg 1993;76(5):10124.

Guyton AC, Hall JE. Textbook of medical physiology. Philadelphia. WB Saunders. 2001; pp 689

Gan TJ, Diemunsch P, Habib AS, Kovac A, Kranke $\mathrm{P}$, Meyer TA, et al. Society for Ambulatory Anesthesia. Consensus guidelines for the management of postoperative nausea and vomiting. Anesth Analg 2014;118(1):85-113.

Gan TJ, Meyer T, Apfel CC, Chung F, Davis PJ, Eubanks $S$ et al. Consensus guidelines for managing postoperative nausea and vomiting. Anesth Analg 2003; 97(1):62-71.

Grabowska-Gaweł A, Porzych K, Piskunowicz G. Risk factors and frequency of postoperative nausea and vomiting in patients operated under general anesthesia. Przegl Lek.2006;63(2):72-6.

Habib AS, Chen YT, Taguchi A, Hu XH, Gan TJ. Postoperative nausea and vomiting following inpatient surgeries in a teaching hospital: a retrospective database analysis. Curr Med Res Opin 2006;22(6):1093-9

Habib AS, Gan TJ. Evidence-based management of postoperative nausea and vomiting: a review. Can J Anaesth 2004;51(4):326-41. 
Henzi I, Sonderegger J, Tramer MR. Efficacy, doseresponse, and adverse effects of droperidol for prevention of postoperative nausea and vomiting. Can J Anaesth 2000;47(6):537-51.

Henzi I, Walder B, Tramer MR. Dexamethasone for the prevention of postoperative nausea and vomiting: a quantitative systematic review. Anesth Analg 2000; 90(1):186-94.

Hickman AG, Bell DM, Preston JC. Acupressure and postoperative nausea and vomiting. AANA J 2005;73(5):379-85.

Huang JC, Shieh JP, Tang CS, Tzeng JI, Chu KS, Wang JJ. Low-dose dexamethasone effectively prevents postoperative nausea and vomiting after ambulatory laparoscopic surgery. Can J Anaesth 2001;48(10):973-77.

Hornby PJ, Central neurocircuitry associated with emesis. Am J Med 2001; 111Suppl (8A): 106S$112 \mathrm{~S}$.

Horn CC, Wallisch WJ, Homanics GE, Williams JP. Pathophysiological and neurochemical mechanisms of postoperative nausea and vomiting. Eur J Pharmacol. 2014. 722: 55-66 doi: 10.1016/j.ejphar.2013.10.037. Epub 2013 Oct 26.

Jokela RM, Cakmakkaya OS, Danzeisen O, Korttila KT, Kranke P, Malhotra A et al., Ondansetron has similar clinical efficacy against both nausea and vomiting. Anaesthesia 2009;64(2):147-51.

Kappen TH, Moons KG, Van Wolfswinkel L, Kalkman CJ, Vergouwe Y, Van Klei WA. Impact of Risk Assessments on Prophylactic Antiemetic Prescription and the Incidence of Postoperative Nausea and Vomiting A Clusterrandomized Trial.Anesthesiology 2014;120(2): 343-54.

Kayhan Z. Clinical Anesthesia. 3.Printing, Logos Publishing, İstanbul 2004;612-5.

Kotani N, Hashimoto H, Sato Y, Sessler DI, Yoshioka H, Kitayama $\mathrm{M}$ et al. Preoperative intradermal acupuncture reduces postoperative pain, nausea and vomiting, analgesic requirement, and sympathoadrenal responses. Anesthesiology 2001;95(2):349-56.

Kovac AL, O'Connor TA, Pearman MH, Kekoler LJ, Edmondson D, Baughman VL et al. Efficacy of repeat intravenous dosing of ondansetron in controlling postoperative nausea and vomiting: A randomized, double-blind, placebo-controlled multicenter trial. J Clin Anesth. 1999;11(6):4539.
Lamond CT, Robinson DL, Boyd JD, Cashman JN. Addition of droperidol to morphine administered by the patient-controlled analgesia method: what is the optimal dose? Eur $\mathrm{J}$ Anaesthesiol 1998;15(3):304-9.

Liao SJ. Acupuncture points: Coincidence with motor points of skeletal muscles. Arch Phys Med Rehabil 1975;56(1):550.

Macario A, Weinger M, Carney S, Kim A. Which clinical anesthesia outcomes are important to avoid? The perspective of patients. Anesth \& Analg 1999;89(3):652 -8.

Macario A, Weinger M, Truong P, Lee M. Which clinical anesthesia outcomes are both common and important to avoid? The perspective of a panel of expert anesthesiologists. Anesth Analg1999;88(5):1085-91.

McCracken G, Houston P, Lefebvre G; Society of Obstetricians and Gynecologists of Canada. Guideline for the management of postoperative nausea and vomiting. J Obstet Gynaecol Can 2008;30(7):600-7, 608-616.

Myklejord DJ, Yao L, Liang H, Glurich I. Consensus guideline adoption for managing postoperative nausea and vomiting. WMJ: official publication of the State Medical Society of Wisconsin 2012; 111(5):207-13 quiz 214.

Melzack R, Stillwell DM, Fox EJ. Trigger points and acupuncture points for pain correlation and implication. Pain 1977;3(1):3-23.

Murphy MJ, Hooper VD, Sullivan E, Clifford T, Apfel CC. Identification of risk factors for postoperative nausea and vomiting in the perianesthesia adult patient. J Perianesth Nurs 2006;21(6):377-84.

Ozatamer O. Current Issues in Anesthesia II. İstanbul 2010;428-31.

Rusy LM, Hoffman GM, Weisman SJ. Electroacupuncture prophylaxis of postoperative nausea and vomiting following pediatric tonsillectomy with or without adenoidectomy. Anesthesiology 2002;96(2):300-5.

Schaub I, Lysakowski C, Elia N, Tramer MR Lowdose droperidol $(\leq 1 \mathrm{mg}$ or $\leq 15 \mu \mathrm{g} \mathrm{kg}-1)$ for the prevention of postoperative nausea and vomiting in adults: quantitative systematic review of randomised controlled trials. Eur J Anaesthesiol 2012;29(6):286-94.

Shenkman Z, Holzman RS, Kim C, Ferrari LR, DiCanzio J, Highfield ES et al. Acupressureacupuncture antiemetic prophylaxis in children undergoing tonsillectomy. Anesthesiology 1999;90(5):1311-6. 


\section{REVIEW}

Tavlan A, Baltaci B, Alptekin A, Ceyhan A, Unal N. A comparison of the effect of single dose ondansetron and P6 (neiguan) acupuncture point on postoperative nausea and vomiting for gynaecological laparoscopy. Eur J Anaesthesiol 1996;13(2):164.

Veiga-Gil L, Pueyo J, and Lopez-Olaondo L. Postoperative nausea and vomiting: physiopathology, risk factors, prophylaxis and treatment. Rev Esp Anestesiol Reanim 2017;64(4): 223-32.

Wang SM, Kain ZN. P6 acupoint injections are as effective as droperidol in controlling early postoperative nausea and vomiting in children. Anesthesiology 2002;97(2):359-66.

Wesmiller SW, Sereika SM, Bender CM, Bovbjerg D, Ahrendt G, Bonaventura M, et al., Exploring the multifactorial nature of postoperative nausea and vomiting in women following surgery for breast cancer. Auton Neurosci. 2017; 202: 10207 doi: 10.1016/j.autneu.2016.09.017. Epub 2016 Sep 28.

White PF, Issioui T, Hu J, Jones SB, Coleman JE, Waddle JP et al. Comparative efficacy of acustimulation (ReliefBand) versus ondansetron (Zofran) in combination with droperidol for preventing nausea and vomiting. Anesthesiology 2002;97(5):1075-81.

Zarate E, Mingus M, White PF, Chiu JW, Scuderi $\mathrm{P}$, Loskota $\mathrm{W}$ et al. The use of transcutaneous acupoint electrical stimulation for preventing nausea and vomiting after laparoscopic surgery. Anesth Analg 2001;92(3):629-35 